\title{
Pertussis Infection in the United States: Role for Vaccination of Adolescents and Adults
}

\author{
Dennis A. Brooks, MD, and Richard Clover, MD
}

Pertussis is the only vaccine-preventable disease on the rise in the United States, with increasing incidence in adolescents and adults related to waning immunity. Although often considered a relatively mild infection in these populations, pertussis can be a serious, potentially deadly illness, particularly in children $<12$ months old. Infected adolescents and adults serve as a reservoir for disease transmission to young children and infants, who are most vulnerable to severe pertussis and its life-threatening complications. In 2005, the US Food and Drug Administration licensed two vaccines that contain tetanus toxoid, reduced diphtheria toxoid and reduced acellular pertussis vaccine (Tdap), one for use in adolescents and the other for use in adolescents and adults. In light of these licensures, the CDC's Advisory Committee on Immunization Practices (ACIP) revised its recommendations for pertussis vaccination to include a Tdap booster dose for all adolescents aged 11 to 12 years as well as catch-up vaccination of those aged 13 to 18 years who did not receive the Td booster. The Committee also recommended a Tdap booster dose for all adults aged 19 to 64 years to replace the next Td booster dose, particularly for those who have close contact with infants $<12$ months old. In February 2006, the ACIP recommended a Tdap booster dose for health care personnel as soon as feasible. If widely administered, Tdap vaccination should have a substantial impact on pertussis. (J Am Board Fam Med 2006;19:603-11.)

Pertussis is a highly contagious, respiratory disease marked by severe and protracted coughing. Its common name, whooping cough, comes from the "whoop" sound often made when affected persons try to inhale during or after a severe coughing spell. Pertussis is caused by Bordetella pertussis, a small

This article was externally peer-reviewed.

Submitted 8 December 2005; revised 18 July 2006; accepted 26 July 2006.

From the Johns Hopkins School of Medicine, Baltimore, MD (DAB); and the University of Louisville School of Medicine, Louisville, KY (RC).

Funding: This study is an outgrowth of an expert roundtable for the Johns Hopkins School of Medicine Advanced Studies in Medicine, sponsored by the Johns Hopkins School of Medicine Office of Continuing Medical Education with support from an unrestricted educational grant from Sanofi Pasteur. At that time, Dr. Brooks was an assistant professor of pediatrics.

Conflict of interest: Dr. Brooks is a Senior Medical Director for Policy, Public Health and Medical Affaires, Merck Vaccine Division, Merck \& Co. The European-based Sanofi Pasteur Merck Sharpe and Dome has an ongoing clinical trial using pertussis combination vaccine (NCT00362427). Dr. Clover has given lectures at Continuing Medical Education-approved events at which the host organization had received unrestricted educational grants from Sanofi Pasteur and Merck.

Corresponding author: Dennis A. Brooks, MD, Merck \& Co., Policy Public Health and Medical Affairs, 770 Sumneytown Pike WP97-B352, West Point, PA 19486 (E-mail: dennis_brooks2@merck.com). aerobic Gram-negative rod first isolated at the beginning of the 20th century. The organism produces multiple antigenic and biologically active substances that play a role in the clinical illness and elicit an immune response conferring immunity. Recent evidence suggests that immunity from $B$. pertussis infection, whether vaccine acquired or natural, is not lifelong. In fact, immunity may wane rather quickly, with protection minimal after 10 years. ${ }^{1}$

Pertussis is associated with substantial morbidity and mortality, especially among the very young and the very old, and imposes an economic burden. About $90 \%$ of pertussis-related deaths are in infants $<6$ months old. ${ }^{2}$ Increased rates of pertussisrelated hospitalizations in persons $>50$ years may be due to increased risk of pneumonia. ${ }^{3}$ Mean medical and nonmedical costs per case in 2002 were $\$ 242$ and $\$ 155$, respectively, for adolescents and $\$ 326$ and \$447, respectively, for adults. ${ }^{4}$ More than $60 \%$ of affected adults miss a mean of 9.8 days from work and over $80 \%$ of affected adolescents miss a mean of 5.5 days from school because of pertussis. ${ }^{4}$

Pertussis remains a public health concern despite availability of an effective diphtheria-tetanus- 


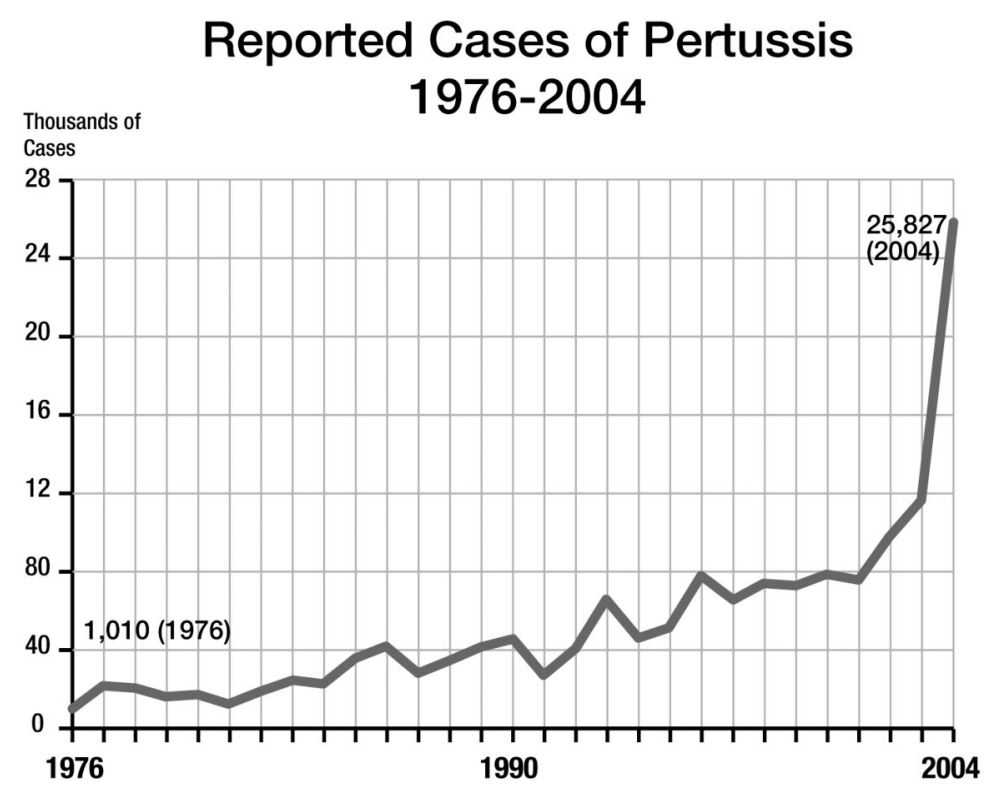

Sources: Centers for Disease Control and Prevention. Historical Summaries of Notifiable Diseases in the United States. MMWR. 52(54); April 22, 2005.

Centers for Disease Control and Prevention. National Immunization Program. Pertussis Surveillance Report, 2004 August 12, 2005

Figure 1. Reported cases of pertussis in the United States, 1976-2004. ${ }^{8,9}$

acellular pertussis (DTaP) vaccine for infants and children. Two pertussis booster vaccines were recently licensed in the United States for use in adolescents; one of the two is also licensed for use in adults. These vaccines may provide an effective method for reducing pertussis incidence, which has been on the rise for decades. The new vaccines combine the tetanus and diphtheria (Td) booster, long recommended for adolescents at 11 to 12 years of age, with acellular pertussis. The resulting booster vaccine (Tdap), therefore, adds no injections to the immunization schedule, while providing much needed protection against this disease. The Centers for Disease Control and Prevention's (CDC) Advisory Committee on Immunization Practices (ACIP) has issued recommendations for Tdap vaccination of adolescents and adults.

\section{Epidemiology and Transmission of Disease}

Pertussis infection is unique to humans. There are no animal reservoirs, and the organism cannot survive for a prolonged period in the environment. Localized to the respiratory tract, the organism is transmitted primarily by aerosol droplets from an infected person to a susceptible one. The infection is highly contagious, with attack rates ranging from $50 \%$ to $100 \%$. The highest attack rates occur among persons with exposure within 5 feet of a coughing patient. ${ }^{5}$

In the pre-vaccine era, pertussis was predominantly an infection of children aged 1 to 5 years, with maternal immunity providing passive protection during an infant's first year of life. At that time, an average of 175,000 US cases were reported per year (incidence of approximately 150 cases per 100,000 population). ${ }^{6}$ The incidence of disease declined steadily over the two decades after introduction of whole-cell pertussis vaccine, reaching an all-time low of just over 1000 reported cases in $1976 .^{7,8}$ Since then, the incidence of infection has continued to rise, with almost 26,000 cases reported to the CDC in 2004 (Figure 1). ${ }^{9}$

Pertussis is the only vaccine-preventable disease on the rise in the US and it is severely underreported. CDC estimates that, at best, one-third of cases are believed to be reported to the $\mathrm{CDC}^{10}$; other estimates place the reported cases at 1 in 10 to 1 in 20 of the true incidence. ${ }^{11}$ Although pertussis incidence remains highest among young infants, rates are also on the rise in adolescents and adults and there may be significant under-reporting in these age groups, especially those with mild or atypical infection. ${ }^{7}$ Compared with surveillance data from 1994 to 1996, the pertussis incidence rate 


\section{Relationship Between Time Since Pertussis Vaccination and Attack Rate}

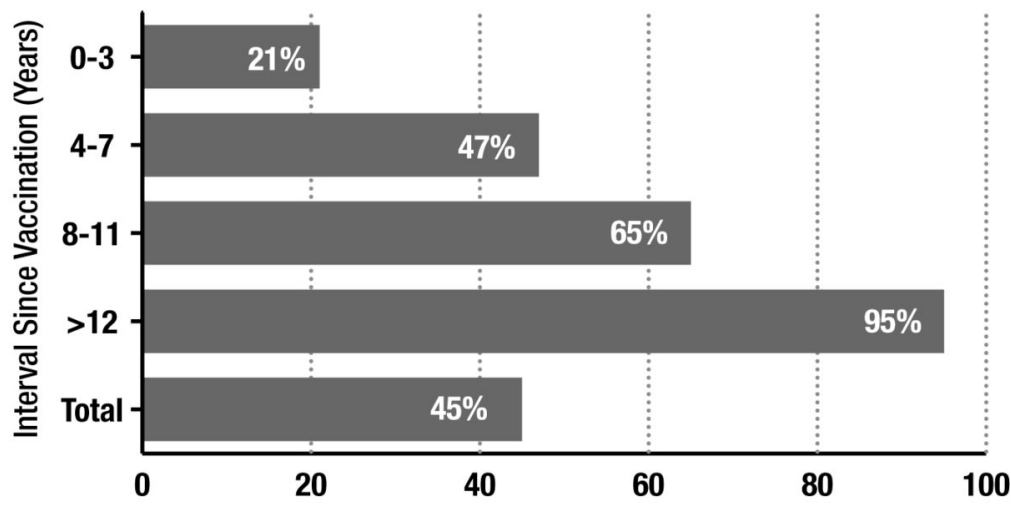

Source: Lambert HJ. Epidemiology of a small Pertussis outbreak in Kent County, Michigan. Public Health Rep. 1965; 80: 365-369.

Figure 2. Relationship between time since pertussis vaccination and attack rate. ${ }^{12}$

among adolescents and adults increased $62 \%$ and $60 \%$, respectively, from 1997 to $2000 .^{7}$

Increased incidence of pertussis in adolescents and adults relates to waning immunity and, likely, to a combination of previous underreporting and recent improvements in reporting processes. The longer the duration since vaccination, the higher the attack rate (Figure 2). ${ }^{12}$ Data from a seroprevalence study by Cattaneo et al showed a peak in antibody titers at 4 to 6 years of age, coinciding with DTaP booster dosing, followed by a decline, and a second peak between 13 and 17 years of age. ${ }^{13}$ Similar findings were documented by the National Health and Nutrition Examination Survey (NHANES), which reported a protracted decline interrupted by a peak in persons aged 40 to 45 years. ${ }^{14}$ Since no pertussis-containing vaccine had been given past the age of 6 years, these spikes in adolescents and older persons clearly represent natural pertussis exposure. These persons not only represent potential cases, but a reservoir of disease that puts those most susceptible to significant morbidity and mortality (ie, those at the extremes of age) at risk of exposure.

Pertussis has been estimated to account for up to $17 \%$ of prolonged cough illness in adults. ${ }^{15,16}$ Among adults with cough illness, the incidence of confirmed pertussis has been estimated at 170 to 630 cases per $100,000 .{ }^{17,18}$ The rates among adolescents were almost 2-fold higher. Data from a prospective acellular pertussis vaccine efficacy trial
(APERT) extrapolate the burden of pertussis to be nearly 1 million US cases annually in persons $\geq 15$ years old. ${ }^{11}$

Given the large disease burden in adolescents and adults estimated by these studies, the limited number of confirmed cases of pertussis (defined in Table 1) in older children, adolescents, and adults is striking. A large proportion of these cases may be atypical and undiagnosed. However, according to recent data from Bisgard et al, ${ }^{19}$ in cases where the source of pertussis was identifiable, adolescents and adults were the primary source of infection for $20 \%$ and $56 \%$, respectively, of infants with pertussis.

Table 1. CDC Definitions of Clinical, Confirmed, and Probable Cases of Pertussis

A clinical case is defined as an acute cough illness lasting $\geq 14$ days in a person with at least one pertussis-associated symptom (ie, paroxysmal cough, post-tussive vomiting without other apparent cause, or inspiratory whoop) or, in an outbreak setting, $\geq 14$ days of cough with no other symptoms.

A confirmed case is defined as a cough illness of any duration in a person from whom $B$. pertussis is isolated, or a case that meets the clinical case definition and is confirmed by polymerase chain reaction (PCR) or by epidemiologic linkage to a culture- or PCR-confirmed case.

A probable case meets the clinical case definition but is neither laboratory confirmed nor epidemiologically linked to a laboratory-confirmed case.

Confirmed and probable cases of pertussis should be reported to the National Notifiable Disease Surveillance System (NNDSS) of the US Centers for Disease Control and Prevention. 
Table 2. Stages of Pertussis Infection ${ }^{24}$

\begin{tabular}{|c|c|c|}
\hline & Duration & Characteristic Signs and Symptoms \\
\hline Incubation period & 7 to 10 days (range, 4 to 21 days) & - \\
\hline \multirow[t]{2}{*}{ Catarrhal stage } & 7 to 14 days & $\begin{array}{l}\text { Insidious onset of coryza, sneezing, and a mild, nonspecific } \\
\text { cough that gradually becomes more severe }\end{array}$ \\
\hline & & In infants: excessive sneezing or 'throat clearing' \\
\hline \multirow[t]{2}{*}{ Paroxysmal stage } & 1 to 6 weeks (up to 10 weeks) & $\begin{array}{l}\text { Bursts, or paroxysms, of numerous, rapid coughs followed } \\
\text { by a long inspiratory effort often associated with a high- } \\
\text { pitched whoop; post-tussive vomiting. }\end{array}$ \\
\hline & & $\begin{array}{l}\text { In infants: paroxysms of coughing, but may not have } \\
\text { sufficient musculature to develop a whoop. In addition, } \\
\text { they may experience gagging, gasping, or eye bulging, } \\
\text { and occasionally bradycardia or cyanosis. }\end{array}$ \\
\hline Convalescent stage & Weeks to months & $\begin{array}{l}\text { Recovery gradual, with cough becoming less paroxysmal } \\
\text { and then disappearing. Fever is generally minimal }\end{array}$ \\
\hline
\end{tabular}

Contact with infected adolescents and adults is a common source of $B$. pertussis infection in infants and unprotected, young children. Widespread silent transmission of pertussis within families has been reported. ${ }^{20}$ In a study of risk factors for pertussis-related hospitalizations, siblings were the most common source (53\%), followed by parents (20\%), other relatives (12\%), neighbors $(8 \%)$, and day-care contacts $(3 \%) .{ }^{21}$ In a case-control study, infants of adolescent mothers (aged 15 to 19 years) were 6-fold more likely to contract pertussis, compared with infants of older mothers (aged 20 to 29 years). ${ }^{22}$

\section{Pathogenesis of Infection}

Details of the pathogenesis of pertussis infection have been extensively reviewed elsewhere. ${ }^{23}$ In brief, the development of pertussis infection begins with entry of $B$. pertussis into the respiratory tract of a susceptible host. The organism produces adhesion and bacterial surface attachment factors that allow its attachment to cilia in the respiratory mucosa. Tracheal cytotoxin and other toxins are produced and released into the local environment, damaging the cilia and respiratory epithelium. These changes disturb clearance of pulmonary secretions and probably result in development of the coryza and cough observed during the catarrhal phase of the illness. Even as local damage increases, attracting host immune cells, the actions of additional toxins probably inhibit phagocyte functions, thereby protecting the proliferating organisms from clearance. In some cases, the proliferation of $B$. pertussis continues until organisms reach the alveoli, resulting in pneumonia.

\section{Clinical Manifestations and Complications of Infection}

Pertussis symptoms are nonspecific in nature, making the clinical diagnosis challenging. The type and severity of symptoms that develop are highly variable, as is the time frame over which they appear and resolve. Adolescents and adults, as well as those partially protected by pertussis vaccine, frequently have mild clinical disease (or even asymptomatic disease) that goes undiagnosed. Although illness may be milder in adolescents and adults, they are a reservoir of infection and may transmit whooping cough to unimmunized or partially immunized infants. Of great concern, health care providers often do not recognize the varied clinical presentations and do not consider a pertussis diagnosis in patients with chronic cough. ${ }^{17}$ Furthermore, management of pertussis is complicated by the fact that infected persons are most contagious early in their illness, before they become symptomatic.

Despite significant interpatient variability, generalizations can be made about the clinical course of illness (Table 2). ${ }^{5,24}$ Pertussis infection develops in four sequential stages, beginning with an incubation period during which infected individuals are asymptomatic followed by three stages of symptomatic illness. The incubation period of pertussis infection commonly lasts for 7 to 10 days, but can be as short as 4 days or as long as 21 days. The first stage in which symptoms can be observed is the catarrhal stage. This stage, which typically lasts 1 to 2 weeks, is characterized by the insidious onset of coryza, sneezing, low-grade fever, and a mild, occasional, nonspecific cough that gradually becomes more severe. In young infants, this stage is often 
characterized by excessive sneezing or "throat clearing."

During the next stage, the paroxysmal stage, many pertussis patients have bursts, or paroxysms, of numerous, rapid coughs, apparently due to impaired mucociliary clearance. At the end of the paroxysm, a long inspiratory effort is oftentimes associated with a high-pitched whoop. Post-tussive vomiting and cyanosis can also occur. The paroxysmal attacks increase in frequency during the first 1 to 2 weeks, remain at the same level for 2 to 3 weeks, and then gradually decrease.

During the convalescent stage, which lasts for weeks to months, recovery is gradual, with cough becoming less paroxysmal and then disappearing.

The symptomatology of infants during the paroxysmal stage of infection is different from that in adolescents and adults. Although very young infants do experience paroxysms of coughing, they often do not "whoop." Although the absent whoop in adolescents and adults is usually associated with milder disease, the whoop may be absent in infants because they lack sufficient musculature in the chest wall to take the deep inhalation that creates the whooping sound. The whoop may appear later in the disease as infants gain in size and strength. Infants may also exhibit clinical symptoms such as gagging, gasping, or eye bulging. Occasionally, they may also present with bradycardia or cyanosis. Pertussis is often ignored in the differential diagnosis of cough illness in young infants due to the absence of a "whoop" and the frequency of concomitant respiratory infections.

\section{Complications of Pertussis}

Life-threatening complications are most common in infants $<3$ months of age, ${ }^{19,21}$ but infection can also be severe in some adult cases. Secondary bacterial pneumonia is diagnosed in up to one quarter of young infants with pertussis ${ }^{21,25}$ and is the most common complication and the cause of most pertussis-related deaths across age groups. Data from 1997 to 2000 indicate that pneumonia occurred in up to $5.2 \%$ of all reported pertussis cases, and $11.8 \%$ of infants $<6$ months of age. ${ }^{7}$ Other complications include seizures $(0.8 \%$ of all cases, $1.4 \%$ of infants $<6$ months of age) and encephalopathy $(0.1 \%$ of all cases, $0.2 \%$ of infants $<6$ months of age).

Death due to pertussis is rare $(0.2 \%)$. The vast majority (90\%) occurs in children younger than 6 months of age with no predisposing conditions. ${ }^{2}$ Risk factors for death among infants include premature birth, Hispanic ethnicity, and having a young mother. ${ }^{2,26}$ Pertussis has also been linked to sudden infant death ( $3 \%$ to $5 \%$ ). ${ }^{27,28}$ Other less severe complications in infants include otitis media, anorexia, and dehydration. Pressure effects of severe paroxysms may lead to pneumothorax, epistaxis, subdural hematomas, hernias, and rectal prolapse. Additional complications identified in adolescents and adults include urinary incontinence, rib fracture, unilateral hearing loss, herniated disk, and precipitation of angina pectoris., ${ }^{5,24}$

\section{Diagnosis of Pertussis}

Laboratory confirmation of pertussis infection is not as straightforward as that of many other infectious diseases. Most local laboratories are not equipped to make the diagnosis of $B$. pertussis infection. The preferred approach is polymerase chain reaction (PCR) testing and a culture of the organism from a posterior nasopharyngeal specimen obtained using two separate Dacron swabs. ${ }^{29}$ For proper collection, the swab must touch the epithelial cells of the posterior nasopharyngeal wall. Culture is the only method from which antibiotic susceptibilities can be measured and molecular typing determined. Isolation of the organism is compromised by recent antibiotic therapy effective against pertussis (ie, macrolide/azalide or trimethoprim-sulfamethoxazole), by delay in specimen collection beyond the first 2 weeks of illness, and in vaccinated persons.

Serologic testing for B. pertussis is limited by a lack of standardization and should not be obtained for clinical decision making. ${ }^{30}$ However, because of its convenience, serology continues to be the most common method used to diagnosis pertussis, especially in the later stages of the infection, making it an important tool in our understanding of disease frequency. ${ }^{31}$ Various enzyme-linked immunosorbent assay (ELISA) techniques are available in commercial laboratories, although there is little evidence of sensitivity or specificity relative to clinical infection. ${ }^{32}$ None of the commercially available serologic tests for pertussis is FDA-licensed for the routine diagnosis of infection. ${ }^{14}$ Direct fluorescent antibody (DFA) is no longer considered useful for diagnosis of pertussis due to low sensitivity and variable specificity. 


\section{Management of Pertussis \\ Antibiotics and Other Agents}

Antibiotic treatment of pertussis infection stops production of pertussis toxin in young infants, which may alter the natural history of the disease. In adolescents and adults, antibiotic therapy must be prescribed early in the disease course to have an impact on duration and severity of symptoms and to lessen the period of communicability. ${ }^{33}$ Close contacts of pertussis cases should be given antibiotics to prevent infection and limit transmission. Prophylactic antibiotics should be initiated as soon as possible within 21 days of exposure. Although not fully efficacious, antibiotic prophylaxis has been shown to be a major factor in controlling household transmission and outbreaks. Although fluids and analgesics may provide some symptom relief, no other therapies (eg, system and aerosolized corticosteroids, beta-agonists, and cough suppressants) have proven beneficial.

\section{Pertussis Vaccination of Children}

DTaP vaccine, which contains purified, inactivated components of $B$. pertussis cells (along with tetanus and diphtheria toxoids), has been available in the United States for more than a decade. Since 1997, the CDC recommends DTaP for all doses of the vaccination series for infants and children $<7$ years of age. The primary immunization series consists of three doses given at 4- to 8-week intervals, beginning at 6 weeks to 2 months of age. A fourth dose is given 6 to 12 months after the third dose. Children who have received all four primary doses before the fourth birthday should receive a fifth (booster) dose before entering school. A fifth dose is not necessary if the fourth dose was administered on or after the fourth birthday.

Point estimates of vaccine efficacy across trials of infants ranged from $80 \%$ to $85 \% .{ }^{24}$ In comparative studies, the acellular pertussis vaccine was significantly more effective than the whole-cell DTP, which is no longer available in the United States. Local and systemic adverse reactions occurred less frequently among infants vaccinated with acellular pertussis vaccine than among those vaccinated with whole-cell pertussis. ${ }^{34}$

\section{Pertussis Vaccination of Adolescents and Adults}

The availability of a less reactogenic acellular pertussis vaccine combined with evidence of substan- tial pertussis infection among adolescents and adults led to reconsideration for the need for acellular pertussis boosters among older subgroups.

Specific formulations of Tdap for adolescents 10 to 18 years, Boostrix ${ }^{\circledR}$ (GlaxoSmithKline), and for adolescents and adults 11 to 64 years, Adacel ${ }^{\circledR}$ (sanofi pasteur), were recently licensed for use in the United States. They contain tetanus toxoid, reduced diphtheria toxoid, and acellular pertussis. In a randomized, controlled, multicenter clinical trial, 10- to 18-year-olds were vaccinated with one dose of Boostrix or a US-licensed $\mathrm{Td}$ vaccine. ${ }^{35}$ Each subject had completed his or her routine childhood vaccinations against diphtheria, tetanus, and pertussis according to the CDC recommended schedule. Boostrix was comparable to the $\mathrm{Td}$ vaccine based on immunogenicity. In both treatment groups, $>99.9 \%$ of subjects had anti-diphtheria and anti-tetanus concentrations greater than 0.1 $\mathrm{IU} / \mathrm{mL}$, indicating seroprotection against these two diseases. In the Boostrix treatment group, antipertussis antibodies levels following primary immunization exceeded (by 1.9 to 7.4 times) those observed in infants, in whom efficacy against pertussis disease was previously demonstrated. The overall safety profile was comparable between the Boostrix and $\mathrm{Td}$ vaccine groups.

Adacel was evaluated in four principal clinical trials in which 7206 individuals (4185 adolescents and 3021 adults) who had not received tetanus or diphtheria toxoid-containing vaccines within 5 years were enrolled. The trials consisted of one randomized, controlled trial that compared Adacel vaccine to a licensed $\mathrm{Td}$ vaccine, ${ }^{36}$ one lot consistency trial, and two concomitant administration trials (one with hepatitis $B$ vaccine and one with influenza vaccine). Across trials, a total of 3393 adolescents and 2448 adults received Adacel vaccine and 792 adolescents and 573 adults received $\mathrm{Td}$ vaccine. In the largest Adacel trial, the seroprotection rate (of at least $0.1 \mathrm{IU} / \mathrm{mL}$ ) for tetanus and diphtheria was $99.8 \%$ and $100 \%$, respectively. ${ }^{36}$ Pertussis antibody geometric mean titers (GMTs) following one dose of Adacel were 2.1- to 5.4-fold higher than those observed among infants following three doses of DTaP. Overall, Adacel vaccine was well tolerated, with local and systemic adverse reactions occurring at similar rates in the Adacel and Td vaccine groups. The ACIP recently revised its recommendations for pertussis vaccination. The immunization schedule now includes a Tdap 
Table 3. DTaP/Tdap Vaccination: Recommendations of the Advisory Committee on Immunization Practices ${ }^{37-39}$

\begin{tabular}{lll}
\hline Dose & \multicolumn{1}{c}{ Age } & Minimum Interval \\
\hline Routine Primary Immunization of Infants & & - \\
1 & 2 months & 4 weeks \\
2 & 4 months & 4 weeks \\
3 & 6 months & 6 months \\
4 & 15 to 18 months* & - \\
5 & 4 to 6 years $\dagger$ & 11 to 12 years \\
Booster dosing of adolescents: & 13 to 19 years $\neq$ & \\
Dosing of Adults $\$$ & 19 to 64 years & \\
All adults who expect to be in close contact & 19 to 64 years \\
with a vulnerable infant &
\end{tabular}

* May be given at 12 months.

$\dagger$ If 4 th dose given before age 4 years.

$\ddagger$ Catch-up vaccination recommended if $\mathrm{Td}$ not received at 11 to 12 years; vaccination with Tdap encouraged for those who received $\mathrm{Td}$ at 11 to 12 years with a 5 -year interval between $\mathrm{Td}$ and Tdap.

$\$$ A single dose of Tdap booster to replace the next scheduled dose of tetanus diphtheria vaccine.

II Infant who has not received 2 to 3 doses of DTaP.

booster dose for all adolescents at 11 to 12 years of age (Table 3). ${ }^{37,38}$ The ACIP also calls for catch-up vaccination of those aged 13 to 18 years who did not receive the Td booster. Finally, because of the importance of controlling pertussis, those in this age range who received the $\mathrm{Td}$ booster are encouraged to get the new Tdap vaccine after a suggested 5 -year interval. This interval may be shortened to as little as 2 years in the presence of increased risk (eg, during outbreaks or periods of increased pertussis activity in the community). ${ }^{7}$

The ACIP also recommends a single dose of Tdap booster to replace the next scheduled dose of tetanus diphtheria vaccine among persons 19 to 64 years of age. In addition, adults who have or who anticipate having close contact with a vulnerable infant (ie, an infant who has not received two to three doses of DTaP) should receive a single dose of Tdap booster. ${ }^{39}$

In February 2006, the ACIP recommended a single dose of Tdap booster as soon as feasible for health care workers in hospital or ambulatory care settings who have direct patient contact. The Committee stated that priority should be given to vaccination of health care workers with direct contact with infants $<12$ months of age. Other health care workers should receive a single dose of Tdap booster according to the routine recommendation and interval guidance for use of Tdap among adults. $^{39}$
Widespread administration of Tdap vaccination should have a substantial impact on pertussis. By way of example, adolescent pertussis immunization programs were recently implemented countrywide in Canada, but started earlier in the Northwest Territories and Newfoundland. Following introduction of an adolescent booster dose, pertussis incidence in the Northwest Territories decreased from 7.9 per 100,000 in the late 1990 s to 0.2 per 100,000 in $2004 .^{40}$ In Newfoundland, no person vaccinated with the Tdap booster has been diagnosed with pertussis to date. ${ }^{41}$

\section{Conclusions}

Pertussis is an often serious, potentially deadly community-acquired illness in persons of all ages. Infected adolescents and adults may suffer substantial morbidity and also are a reservoir for disease transmission to infants. Newly available Tdap vaccines are safe and effective in preventing infection. The addition of a pertussis booster to the previously available tetanus and diphtheria booster will not only directly benefit vaccine recipients but may allow for greater control of the pertussis reservoir in adolescents and adults, potentially leading to decreased incidence in infants who are at highest risk for severe complications including death. If widely administered, Tdap vaccination should have a substantial impact on pertussis. 
At the time he cowrote this manuscript, Author 1 was assistant professor of pediatrics at Johns Hopkins School of Medicine.

\section{References}

1. Jenkinson D. Duration of effectiveness of pertussis vaccine: evidence from 10-year community study. BMJ 1988;296:612-4.

2. Mikelova LK, Halperin SA, Scheifele D, et al. Predictors of death in infants hospitalized with pertussis: a case-control study of 16 pertussis deaths in Canada. J Pediatr 2003;143:576-81.

3. De Serres G, Shadami R, Duval B, et al. Morbidity of pertussis in adolescents and adults. J Infect Dis 2000;182:174-9.

4. Lee GM, Lett S, Schauer S, et al. Societal costs and morbidity of pertussis in adolescents and adults. Clin Infect Dis 2004;39:1572-80.

5. Hewlett EL. Bordetella species. In: Principles and Practice of Infectious Diseases. 5th ed. (Mandel GL, Bennett JE, Dolin R, eds). Philadelphia, PA: Churchill Livingstone; 2000, p. 2414-22.

6. Cherry JD. Pertussis in preantibiotic and prevaccine era, with an emphasis on adult pertussis. Clin Infect Dis 1999;28:S107-S11.

7. Centers for Disease Control and Prevention. Pertussis: United States, 1997-2000. MMWR 2002;51:73-6.

8. Centers for Disease Control and Prevention. Historical summaries of notifiable diseases in the United States. MMWR 2005;52.

9. Centers for Disease Control and Prevention. National Immunization Program. Pertussis Surveillance Report, 2004 (Final). August 12, 2005. Unpublished data.

10. Sutter RW, Cochi SL. Pertussis hospitalizations and mortality in the United States, 1985-1988. Evaluation of the completeness of national reporting. JAMA 1992;267:386-91.

11. Ward JI, Cherry JD, Chang S-J, et al. Efficacy of an acellular pertussis vaccine among adolescents and adults. N Engl J Med 2005;353:1555-63.

12. Lambert HJ. Epidemiology of a small pertussis outbreak in Kent County, Michigan. Public Health Rep 1965;80:365-9.

13. Cattaneo LA, Reed GW, Haase DH, Wills MJ, Edwards KM. The seroepidemiology of Bordatella pertussis infections: a study of persons ages 1-65 years. J Infect Dis 1996;173:1256-9.

14. Baughman AL, Bisgard KM, Edwards KM, et al. Establishment of diagnostic cutoff points for levels of serum antibodies to pertussis toxin, filamentous hemagglutinin, and fimbriae in adolescents and adults in the United States. Clin Diagn Lab Immunol 2004;11:1045-53.

15. Cherry JD. The epidemiology of pertussis: a comparison of the epidemiology of the disease pertussis with the epidemiology of Bordetella pertussis infection. Pediatrics 2005;115:1422-7.
16. Jackson LA, Cherry JD, Wang SP, Grayston ST. Frequency of serological evidence of Bordetella infections and mixed infections with other respiratory pathogens in university students with cough illnesses. Clin Infect Dis 2000;31:3-6.

17. Nennig ME, Shinefield HR, Edwards KM, Black SB, Fireman BH. Prevalence and incidence of adult pertussis in an urban population. JAMA 1996;275: 1672-4.

18. Strebel P, Nordin J, Edwards K, et al. Populationbased incidence of pertussis among adolescents and adults, Minnesota, 1995-1996. J Infect Dis 2001; 183:1353-9.

19. Bisgard KM, Pascual FB, Ehresmann KR, et al. Infant pertussis: who was the source?. Pediatr Infect Dis J 2004;23:985-9.

20. Long SS, Welkon CJ, Clark JL. Widespread silent transmission of pertussis in families: Antibody correlates of infection and symptomatology. J Infect Dis 1990;161:473-9.

21. Halperin SA, Wang EE, Law B, et al. Epidemiological features of pertussis in hospitalized patients in Canada, 1991-1997: Report of the Immunization Monitoring Program: Active (IMPACT). Clin Infect Dis 1999;28:1238-43.

22. Izurieta HS, Kenyon TA, Strebel PM, et al. Risk factors for pertussis in young infants during an outbreak in Chicago in 1993. Clin Infect Dis 1996;22: 503-7.

23. Hewlett EL. Pertussis: current concepts of pathogenesis and prevention. Pediatr Infect Dis J 1997;16: S78-S84.

24. Centers for Disease Control and Prevention. Epidemiology and Prevention of Vaccine-Preventable Diseases. 9th ed.; 2006.

25. Tanaka M, Vitek CR, Pascual FB, et al. Trends in pertussis among infants in the United States, 19801999. JAMA 2003;290:2968-75.

26. Vitek CR, Pascual FB, Baughman AL, Murphy TV. Increase in deaths from pertussis among young infants in the United States in the 1990s. Pediatr Infect Dis J 2003;22:628-34.

27. Lindgren C, Milerad J, Lagercrantz H. Sudden infant death and prevalence of whooping cough in the Swedish and Norwegian communities. Eur J Pediatr 1997;156:405-9.

28. Heininger U, Kleemann WJ, Cherry JD, et al. A controlled study of the relationship between bordetella pertussis infections and sudden unexpected deaths among German infants. Pediatrics 2004;114: $9-15$.

29. Brooks DA, Edwards KM, Long SS, et al. The Rise of Adolescent Pertussis in the United States. Adv Stud Med 2005;5:S450-S452.

30. Centers for Disease Control and Prevention. Recommended antimicrobial agents for treatment and postexposure prophylaxis of pertussis: 2005 CDC Guidelines. MMWR 2005;54:1-16. 
31. Tozzi AE, Celentano LP, Ciofi degli Atti ML, et al. Diagnosis and management of pertussis. CMAJ 2005;172:509-15.

32. Cherry JD, Grimprel E, Guiso N, Heininger U, Mertsola J. Defining pertussis epidemiology: clinical, microbiologic, and serologic perspectives. Ped Infect Dis J 2005;24:S25-S34.

33. Bortolussi R, Miller B, Ledwith M, et al. Clinical course of pertussis in immunized children. Ped Infect Dis J 1995;14:870-874.

34. Pines E, Barrand M, Fabre P, et al. New acellular pertussis-containing paediatric combined vaccines. Vaccine 1999; 17:1650-6.

35. Boostrix ${ }^{\circledR}$ Prescribing Information. GlaxoSmithKline; 2005.

36. Pichichero ME, Rennels MB, Edwards KM, et al. Combined tetanus, diphtheria, and 5-component pertussis vaccine for use in adolescents and adults. JAMA 2005;293:3003-11.
37. Centers for Disease Control and Prevention. ACIP recommends adolescent vaccination for tetanus, diphtheria and pertussis vaccine, June 30, 2005. Available at: www.cdc.gov/nip/pr/pr_tdap_jun2005.htm

38. Centers for Disease Control and Prevention. Pertussis vaccination: use of acellular pertussis vaccines among infants and young children. MMWR 1997; 46:1-25.

39. Centers for Disease Control and Prevention. ACIP votes to recommend use of combined tetanus, diphtheria, and pertussis (Tdap) vaccine for adults, March 2, 2006. Available at: www.cdc.gov/nip/vaccine/tdap/tdap_adult_recs.pdf

40. Kandola K, Lea A, Santos M. Pertussis rates in Northwest Territories after introducing adult formulation acellular vaccine (abstract). Can J Infect Dis Med Microbiol 2004;15:351.

41. Pertussis in Newfoundland and Labrador: 1991-2004. CCDR 2005;31:235-7. 